\title{
Conservación de la naturaleza en propiedad privada: las Reservas Naturales de la Sociedad Civil en el Valle del Cauca*
}

\author{
Nature conservation on private land: \\ Natural Reserves of Civil Society in \\ Valle del Cauca
}

\author{
Conservação da natureza em \\ propriedade privada: As Reservas \\ Naturais da Sociedade Civil no \\ Valle del Cauca
}

\footnotetext{
* Una versión completa de esta investigación corresponde al trabajo de grado en Economía de la Universidad del Valle, de Melissa Quintero López dirigido por Fabio A. Arias.

** Economista, investigadora de la Asociación para el Estudio y Conservación de las Aves Acuáticas en Colombia, Calidris, Cali, Colombia, Dirección postal: Cra 60a No. 2a -79, Cali, Colombia. Correo electrónico melissa.quintero. lopez@gmail.com

*** Economista, Ph.D en Desarrollo Sustentable, profesor de la Universidad del Valle, Cali, Colombia. A.A. 25360, Cali, Colombia. Correo electrónico; fabio.arias@correounivalle.edu.co
} 


\section{Resumen}

Las Reservas Naturales de la Sociedad Civil en Colombia cumplen un papel importante en la conservación de ecosistemas. En este trabajo se investiga por qué los dueños de áreas privadas en el departamento del Valle del Cauca conservan la naturaleza en sus propiedades. Se consideran las explicaciones entre la teoría económica de la elección racional, la teoría económica sobre los comportamientos altruistas y recíprocos y la teoría de la psicología social sobre los valores de orientación cultural. Se encuentra que las razones predominantes para la conformación de las reservas naturales son el interés propio y la valoración de la naturaleza por parte de sus dueños.

Palabras clave: conservación de la naturaleza, Reservas Naturales de la Sociedad Civil, incentivos, elección racional, altruismo, valores de orientación cultural.

Clasificación JEL: Q21, Q24.

\section{Abstract}

Natural Reserves of the Civil Society in Colombia plays an important role in the conservation of ecosystems. This paper investigates why the owners of private areas in Valle del Cauca preserve nature in their properties. Explanations among economic rational choice theory, economic theory on reciprocal altruistic behavior and the theory of social psychology on the values of cultural orientation are considered. We found the predominant reasons for the establishment of nature reserves are self-interest and appreciation of the nature by land owners.

Keywords: conservation of nature, Natural Reserves of Civil Society, incentives, rational choice, altruism, cultural values orientation. 


\section{Resumo}

As Reservas Naturais da Sociedade Civil na Colômbia têm um importante papel na conservação dos ecossistemas. Este artigo investiga por que os proprietários de áreas privadas, no estado do Valle del Cauca, preservam a natureza em suas propriedades. Explicações entre a teoria econômica da escolha racional, teoria econômica sobre o comportamento altruísta ou recíproco e a teoria da psicologia social sobre os valores de orientação cultural são consideradas. As razões predominantes para a criação de reservas naturais são o auto-interesse e a apreciação da natureza de seus proprietários.

Palavras chave: Conservação da Natureza, Reservas Naturais da Sociedade Civil, incentivos, escolha racional, o altruísmo, valores de orientação cultural. 


\section{INTRODUCCIÓN}

En Colombia existen diferentes mecanismos de conservación privada de la naturaleza, pero el único que tiene reconocimiento legal son las Reservas Naturales de la Sociedad Civil. Esta figura legal nace de las exigencias e iniciativas de propietarios que conservaban de forma privada en sus propiedades y que demandaron el reconocimiento de sus acciones por parte de la nación. Las Reservas Naturales de la Sociedad Civil son entonces propiedades privadas donde se realizan acciones de conservación y producción sostenible, que por voluntad del propietario fueron registradas ante la Unidad Administrativa Especial del Sistema de Parques Nacionales Naturales de Colombia, unidad encargada del manejo de todas las áreas protegidas del país (tanto públicas como privadas). Los propietarios de las reservas realizan una importante labor complementaria a la del Estado, la cual es de gran importancia para la conservación de recursos que el Gobierno no está garantizando.

Como en la mayoría de países, en Colombia, buena parte del territorio se encuentra en manos de propietarios privados, por ello, la conservación privada es importante. No todos los propietarios de predios deciden conservar, pues los usos que le dan a sus propiedades son diversos. Las diferentes alternativas de uso que le pueden dar a su propiedad, el hecho de que los propietarios de las Reservas Naturales de la Sociedad Civil decidieron registrar su propiedad ante el Estado, y la necesidad de más áreas de conservación privadas, hace relevante analizar esta situación. En este trabajo se indaga por qué los propietarios de predios privados decidieron conservar la naturaleza en sus propiedades y las conformaron como una Reserva Natural de la Sociedad Civil. 
Por diversas limitaciones no se puede abarcar todo el país, Colombia cuenta con Reservas Naturales de la Sociedad Civil en la gran mayoría de departamentos. En este trabajo se eligió realizar la investigación en el departamento del Valle del Cauca, que es uno de los departamentos pioneros en conservación. En el Valle del Cauca hay más de sesenta Reservas Naturales de la Sociedad Civil registradas y existen muchas propiedades donde se realizan labores de conservación y producción sostenible que no han sido inscritas. El departamento también cuenta con varias organizaciones no gubernamentales que velan por la conservación, que apoyan a los propietarios de las reservas para la implementación de proyectos en pro de los objetivos de protección del medio ambiente y producción sostenible.

No existe una teoría que brinde una explicación única al por qué las personas deciden conservar en sus propiedades y conformar una Reserva Natural de la Sociedad Civil. Por lo tanto, se exploran los incentivos que existen en Colombia para conservar de forma privada y se plantean también tres líneas teóricas que brindan diferentes explicaciones para dicha decisión. La primera línea teórica planteada es la teoría económica sobre la elección racional, la segunda es la teoría económica sobre la provisión privada del bien público y los comportamientos no autointeresados, la última línea o perspectiva considerada proviene de la psicología social, y es la teoría sobre los valores de orientación cultural.

El enfoque metodológico de la investigación es explicativo y deductivo. A partir de las teorías exploradas en el marco teórico y la información sobre derechos e incentivos de las Reservas Naturales de la Sociedad Civil fueron propuestas unas hipótesis, posteriormente contrastadas con la información recopilada. De tal manera, que la investigación también involucra un enfoque empírico. Para la recopilación de información se eligió una muestra de Reservas Naturales de la Sociedad Civil en el Valle del Cauca de acuerdo con el criterio de saturación. El instrumento elegido para recopilar la información es la entrevista semiestructurada. Finalmente se presentan los resultados, se esboza un análisis de la información recopilada que pretende contrastar las hipótesis formuladas.

\section{ANTECEDENTE DE LA CON- SERVACIÓN PRIVADA DE LA NATURALEZA}

A continuación se presenta el objeto y área de estudio de esta investigación: las Reservas Naturales de la Sociedad Civil en el departamento del Valle del Cauca. Como acercamiento al objeto, se presenta una breve ilustración de la conservación privada en América, se presenta una ilustración de la conservación privada en Colombia; se ilustra cómo nace la figura legal de Reserva Natural de la Sociedad Civil y 
la importancia de estas áreas para el país. Posteriormente se presenta el área de estudio, el departamento del Valle del Cauca, sus condiciones ambientales y las Reservas Naturales de la Sociedad Civil que hay en el departamento. Finalmente se abordan los incentivos y derechos que tienen los propietarios de las Reservas Naturales de la Sociedad Civil para conservar en sus propiedades.

\section{Conservación privada en América}

La conservación privada en América es una figura que ha cogido gran fuerza como alternativa de conservación de la naturaleza. La insuficiencia de áreas protegidas públicas para detener la degradación y el creciente interés de la sociedad civil de participar en la conservación de la biodiversidad, han influenciado el crecimiento de áreas de conservación privada en la región. Según Chacón (2008), este interés de la sociedad civil de participar en la conservación de la naturaleza, no es reciente, hace parte de las prácticas sostenibles tradicionales de los pueblos. Sin embargo, según Monteferri y Coll (2009), en América la conservación privada como figura surge inicialmente en el norte, con la conservación de tierras privadas bajo el nombre de contratos de servidumbre, de forma voluntaria, por parte de los propietarios de estas tierras quienes buscaban limitar los derechos de explotación y desarrollo en sus propiedades. En la actualidad existen propietarios individuales y comunitarios, redes y sistemas de conservación en la mayoría de países americanos.

Monteferri y Coll (2009) señalan que en Latinoamérica las iniciativas de conservación de la naturaleza han sido abordadas e implementadas bajo diferentes figuras. Han sido implementadas de forma voluntaria como también impositiva, con apoyo del Estado o sin este, y con el apoyo en algunos casos de diversas ONG. Según estos autores, Brasil es el país pionero en incluir esta figura como una alternativa de conservación en su legislación, cuenta con un fuerte respaldo de la sociedad civil y más de 1500 áreas protegidas de propietarios privados, reconocidas por el Gobierno. Colombia también cuenta con un gran número de áreas y una figura legal reconocida por el Estado. En Venezuela, Ecuador, Bolivia y Perú, el apoyo del Estado y el respaldo de la sociedad civil ha sido poco, pero se han logrado desarrollar pequeños sistemas de conservación significativos. En la Guyana Francesa la conservación en tierras privadas ha sido impuesta por el Estado.

\section{Conservación privada en Colombia}

La conservación privada en Colombia surge a partir de iniciativas de propietarios que de forma voluntaria deciden conservar la naturaleza e implementar prácticas sostenibles de producción en sus predios. Hace más de dos décadas, con el incremento de predios privados de conservación y la organización colectiva de algunos de estos propietarios 
en una red denominada Resnatur, se planteó la necesidad de una figura legal que amparara las iniciativas de conservación. Con la Ley 99 de 1993, los propietarios pertenecientes a la red Resnatur lograron que el Estado implementara una figura legal para los predios privados que conservan la naturaleza. La categoría jurídica implementada por el Estado es la Reserva Natural de la Sociedad Civil (RNSC), definida como: “... la parte o el todo del área de un inmueble que conserve una muestra de un ecosistema natural y sea manejado bajo los principios de la sustentabilidad en el uso de los recursos naturales" (Ley 99 de 1993, art. 109).

Ocampo (2010) destaca que con la categoría jurídica de RNSC se buscaba que el Estado reconociera el aporte de la sociedad civil a la conservación y apoyara estas iniciativas.

Escobar y Solano (2009) señalan que los modelos de conservación y funciones varían entre reservas de acuerdo con los actores involucrados y sus intereses. Según estos autores, las reservas pueden ser propiedad de una ONG, familias, empresas, o pueden ser comunitarias, además de dedicarse a la conservación, las reservas pueden realizar otras actividades como ecoturismo, educación ambiental, sistemas de producción sostenible, investigación, y habitación permanente. En Colombia la institución encargada de las áreas protegidas tanto privadas como públicas es Parques Nacionales
Naturales de Colombia. Según la información de esta entidad, disponible en su página web, el número de RNSC registradas en todo el país hasta el año 2011 era 275.

\section{Área de estudio: el departamento del Valle del Cauca, Colombia}

En el departamento del Valle del Cauca han sido registradas 63 Reservas Naturales de la Sociedad Civil hasta el año 2014 (CVC, 2014a). En el departamento existen, además, áreas de conservación privada que aún no han sido registradas o que están en trámite, por lo que se estima que las áreas de conservación son muchas más. En 14 municipios del departamento hay RNSC, siendo Versalles, El Cairo y Dagua los municipios con mayor número de reservas, mientras que Cali, Sevilla y Palmira, los municipios con el menor número de reservas. En la Figura 1 se puede observar el número de RNSC por municipio.

Las características, funciones y objetivos de conservación, varían en cada reserva de acuerdo con sus condiciones geográficas y los intereses de sus propietarios. En el departamento se pueden encontrar reservas desde las 0,16 ha en el municipio de Bolívar, hasta de 269,838 ha en el municipio de Dagua. Los municipios con mayor número de hectáreas en total conservadas de forma privada (suma de las hectáreas de las RNSC registradas en el municipio), son Dagua, Tuluá, El 


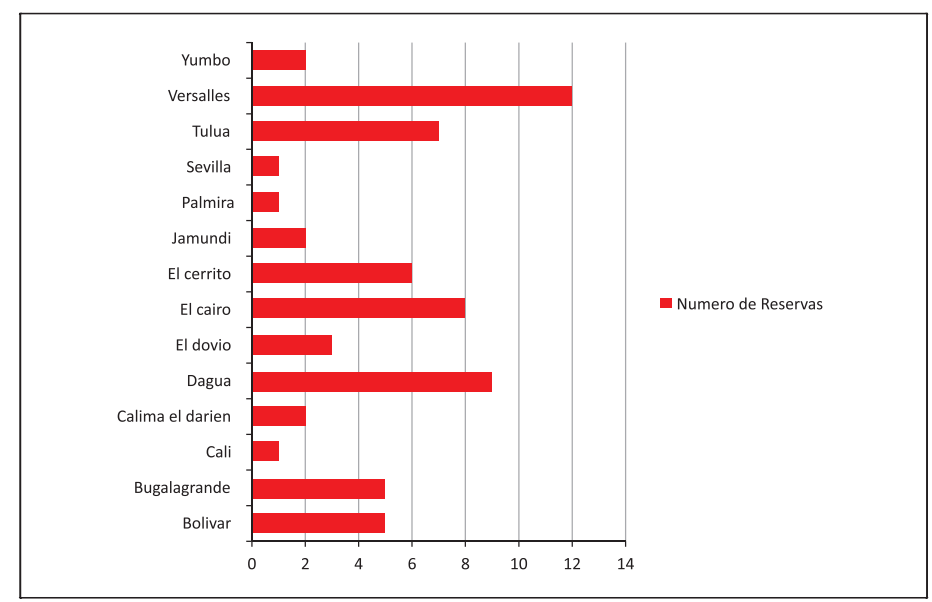

Figura 1. RNSC registradas por municipio

Fuente: elaboración propia con base en datos suministrados por Parques Nacionales Naturales de Colombia (2014a, 2014b, 2014c)

Cerrito y Versalles. En la Figura 2 se puede observar el total de hectáreas conservadas por municipio. Los objetivos de conservación, por lo general, consisten en conservar el ecosistema natural presente dentro de la reserva, aunque algunas reservas especifican la protección de alguna especie vegetal o animal que sea característica del área y que se encuentre amenazada.

El ecosistema presente en la mayoría de RNSC del departamento es la selva andina y subandina. En municipios como Tuluá, El Cerrito, El Cairo, Bugalagrande, Versalles, Calima, Yumbo, entre otros, se puede encontrar este tipo de ecosistema. En el municipio de Dagua se encuentra un ecosistema muy especial, el enclave subxerofítico, por ello, las reservas ubicadas en este municipio cobran gran importancia en la protección de este ecosistema único de la zona andina. Uno de los mayores aportes de las RNSC es la protección de la oferta hídrica de la región, pues la mayoría de RNSC protege nacimientos, quebradas y vertientes.

\section{Derechos e incentivos de las Reservas Naturales de la Sociedad Civil}

En el Decreto 1996 de 1999, que reglamenta los artículos 109 y 110 de la Ley 99 de 1993 sobre Reservas Naturales de la Sociedad Civil, se establecen los derechos que adquieren los propietarios al conformar su predio como una RNSC. Los derechos son: derechos de participación en los procesos de planeación de programas de desarrollo, derecho al consentimiento 




Figura 2. Número de hectáreas de conservación privada (RNSC) en cada municipio Fuente: elaboración propia con base en datos suministrados por la CVC (2014b)

previo para la ejecución de inversiones públicas que afecten la RNSC y derecho a incentivos. Sobre los incentivos el decreto establece que tanto el Gobierno nacional como las entidades territoriales, deberán crear incentivos dirigidos a la conservación por parte de los propietarios de las RNSC (Decreto 1996, art 11, 12, 13,14).

Escobar y Solano (2009) afirman que, a nivel nacional y municipal, se han intentado formular diferentes tipos de incentivos que no se han puesto en práctica. El único incentivo que han logrado implementar algunas RNSC, según estas autoras, es la exención del impuesto predial, que es solicitado ante el municipio donde se encuentre ubicada la reserva y depende de este su otorgamiento. No obstante, a nivel regional, la CVC (2012), también ha aplicado incentivos para las RNSC del Valle del Cauca, realizando inversiones para el desarrollo de diferentes proyectos que beneficien las reservas, como: apoyo a la formulación de sus planes de manejo, reconversión agroecológica, sistemas silvopastoriles, investigación, reforestación, mercados verdes, etc.

La decisión de conservar de forma privada es beneficiosa para el país, sin embargo, no todos los propietarios 
privados deciden conservar. En este sentido, los propietarios de las RNSC tomaron una decisión muy particular; conservar en sus propiedades frente a diversos usos alternativos. Dichos propietarios debieron haber tenido algunos incentivos o motivaciones que los llevaron a tomar esta decisión y mantener la conservación en sus predios. ¿Por qué conservan áreas naturales en sus propiedades? ¿Por qué registraron su propiedad como una Reserva Natural de la Sociedad Civil? ¿Por qué estos agentes no dan otro uso a su propiedad? ¿Existen incentivos para la conservación privada de la naturaleza en el departamento del Valle del Cauca y Colombia? ¿Cuáles son las motivaciones que llevaron a estos agentes a conservar en sus propiedades?

\section{MARCO TEÓRICO}

No se plantea una única forma de abordar el tema, es decir, una única teoría, ya que ninguna de estas brinda una explicación completa del porqué los individuos deciden conservar. De las líneas teóricas contempladas, dos pertenecen al campo de investigación económico y una pertenece al campo de investigación de la psicología social. La primera línea teórica contemplada es la teoría económica de la elección racional, esta teoría plantea que los individuos conservarían la naturaleza porque ello les brinda utilidad individual. La segunda línea es la teoría económica sobre los comportamientos no autointeresados, las decisiones de los individuos pueden no solo buscar el interés personal. En esta línea también se abarca la provisión privada del bien público, acciones altruistas, recíprocas, entre otras, que pueden considerarse como donaciones o contribuciones voluntarias al bien público. La última línea teórica contemplada proviene de la psicología social, la teoría sobre los valores de orientación cultural propuesta por Schwartz (2006, 2007). Esta teoría propone que las sociedades tienen unos determinados valores de orientación cultural que explican el comportamiento individual.

\section{Agentes racionales y egoístas}

A partir de la teoría de la elección racional (Pindyck \& Rubinfeld, 2013; Varian, 1993) se puede considerar la decisión de conservar como una decisión de consumo, como un problema de maximización de la utilidad individual (Field \& Azqueta, 1996; Kolstad, 2000; Hanley \& Barbier, 2009). Bajo esta perspectiva, conservar la naturaleza puede analizarse como una elección de consumo, es decir, los individuos en su cesta de consumo además de consumir los bienes comunes, consumen un bien denominado naturaleza. La naturaleza, como los demás bienes, le proporciona una utilidad al individuo y es preferida ante otras alternativas. La conservación de la naturaleza corresponde entonces a una decisión en pro del interés individual. Este enfoque plantea algunos interrogantes: ¿Consideran los individuos que deciden conservar la naturaleza 
en sus propiedades esta elección como una decisión de consumo? ¿Constituye la naturaleza un bien más del cual estos individuos obtienen utilidad?

Desde una perspectiva de la elección racional, Hardin (1968) y Olson (1999) proponen la incapacidad o dificultad de conseguir un acuerdo social para alcanzar un bienestar común partiendo de intereses individuales. Por su parte, en Ostrom (2000) y Ostrom et al. (2002), se constata que tal coordinación es posible por medio de la formulación y puesta en práctica de reglas que regulan el uso y la interacción entre los miembros de las comunidades. Se consideró importante mencionar de forma rápida que la teoría de la elección racional ha estudiado ampliamente los bienes de uso común. Sin embargo, las líneas de estudio sobre bienes ambientales mencionadas no plantean una manera de analizar el porqué los individuos conservan áreas naturales en sus propiedades.

\section{Más allá del supuesto de individuos autointeresados}

Conservar la naturaleza en propiedades privadas puede no solo corresponder a una decisión de autointerés. Puede involucrar una preocupación por el bienestar de otros, es decir, una decisión que busca beneficiar no solo al individuo, sino a otras personas o la comunidad. Las acciones, decisiones o comportamientos no autointeresados, no son analizados en el modelo económico de la elección racional, sin embargo, desde hace un par de décadas algunos investigadores en este campo se han interesado en modelarlos y estudiarlos. Los diferentes análisis planteados proponen como comportamientos no autointeresados, los comportamientos altruistas, recíprocos, prosociales, entre otros.

Las interacciones sociales influyen los comportamientos de las personas y sus decisiones. Becker (1974) fue uno de los pioneros en intentar incorporar comportamientos no egoístas, o lo que él denominó interacciones sociales, en la teoría económica moderna. Según este autor, las características personales de otros individuos afectan la utilidad y el ingreso, por ejemplo, las buenas acciones de una persona incentivan a otras a comportarse de la misma manera. Trabajos más recientes analizan los comportamientos no egoístas como un tipo de preferencias denominadas "preferencias sociales", que en su versión más sencilla son descritas como un interés individual por el bienestar de otros individuos además del interés propio, en este sentido, las preferencias sociales según Cabrales y Ponti (2011) son interdependientes; los individuos tienen en cuenta además de sus propias preferencias las preferencias de otros.

Fehr y Fischbacher (2002) plantean que existen diversos tipos de preferencias sociales; las preferencias recíprocas, el altruismo puro, la aversión a la desigualdad, y la envidia. Las 
preferencias recíprocas describen comportamientos individuales de acuerdo con las acciones de otros individuos, es decir, un individuo con preferencias recíprocas responderá de manera amable ante acciones amables y de manera hostil ante acciones hostiles. Las acciones son clasificadas como hostiles o amables dependiendo de la justicia o injusticia de sus consecuencias o de la intención que las motivó. El altruismo puro es un tipo de preferencia social que describe el comportamiento de individuos que valoran de manera positiva los recursos o ganancias de otros agentes, y nunca ejecutan acciones en contra de estas ganancias. El altruismo puro, a diferencia de la reciprocidad, no surge ante acciones altruistas, es incondicional.

La teoría sobre las preferencias sociales, resumiendo, propone que los individuos tienen en cuenta las preferencias y pagos de otros individuos. La teoría sobre las interacciones sociales plantea que los individuos tienen en cuenta las características de otros individuos; ambas teorías proponen que los individuos no solo tienen preferencias egoístas. Para el objeto de este trabajo podría entonces preguntarse, si los individuos que tomaron la elección de conservar en sus territorios tienen preferencias sociales, o puede asumirse que se preocupan por el bienestar o características de otros individuos. En el caso de que estos individuos exhibieran preferencias sociales, ¿qué tipo de preferencias sociales exhiben? ¿Exhiben preferencias sociales recíprocas?

Fehr y Fischbacher (2002) no son los primeros en plantear que los individuos pueden comportarse por motivaciones recíprocas o altruistas, otros economistas han analizado estas motivaciones. Un determinante fundamental del comportamiento humano es la reciprocidad. Zamagni (2006) plantea que la motivación recíproca es una respuesta comportamental a la cortesía percibida, de tal manera que las transferencias entre individuos son bidireccionales pero voluntarias y no existen perspectivas de ganancias. El altruismo puro, a diferencia de la reciprocidad, no espera transferencias bidireccionales. En especial, este autor se ha interesado por los comportamientos individuales que benefician las agrupaciones sociales y comunidades, comportamientos que denomina como prosociales (Zamagni, 1995).

Pena y Sánchez (2006) subrayan que los comportamientos prosociales descritos por Zamagni pueden originarse de comportamientos altruistas y de comportamientos no altruistas. Los comportamientos altruistas pueden ser motivados por la simpatía o el deber, mientras que los comportamientos no altruistas pueden estar motivados por el reconocimiento social y redes de aseguramiento, o por instituciones de cooperación no voluntaria. En el caso de que el altruismo sea motivado por 
el deber, los individuos son animados por valores morales.

Los trabajos citados plantean varios aspectos considerables; en primer lugar, reconocen la importancia y necesidad de asumir individuos con motivaciones diferentes a las que asume el modelo económico de la elección racional, es decir, al egoísmo o autointerés. En segundo lugar, plantean que estas motivaciones obedecen a preocupaciones por el bienestar de otros individuos o comportamientos morales. En tercer lugar, coinciden en que esas motivaciones son derivadas del altruismo o la reciprocidad. En cuarto lugar, tanto la teoría de las interacciones sociales como la teoría de los comportamientos prosociales analizan el caso de las donaciones y las posibles motivaciones detrás de ellas. Identificados estos aspectos claves, surge un nuevo interrogante: ¿Qué tanto puede explicar esta línea teórica la elección de conservar la naturaleza en territorios privados? ¿Puede caracterizarse la decisión conservación privada como un comportamiento prosocial?

\section{Provisión privada de bienes públicos}

Una línea de investigación que complementa la anterior es la provisión privada de bienes públicos. En el campo económico se han estudiado las donaciones y contribuciones voluntarias como provisión privada del bien público. Las donaciones y contribuciones voluntarias se caracterizan, por lo general, por no ser excluyentes, la contribución de una persona confiere beneficios a un grupo, por esta razón, Sugden (1984) argumenta que los bienes provistos por el sector voluntario pueden catalogarse como bienes públicos. La conservación de la naturaleza puede ser catalogada como una labor que confiere beneficios a la sociedad, ya que el mantenimiento, por ejemplo, de bosques, ayuda a mantener el aire limpio, y de agua, a mantener fuentes hídricas. Desde esta perspectiva se puede plantear que conservar la naturaleza puede verse como una contribución al bien público. En esta sección se retoman algunos trabajos sobre este tema.

La mayoría de trabajos sobre provisión privada de bienes públicos, analizan la eficiencia de dicha provisión o las motivaciones por las cuales los individuos contribuyen. Un trabajo muy citado es el de Bergstrom, Blume y Varian (1986); estos autores analizan bajo un modelo general la provisión privada de un bien público, en el que los individuos tienen preferencias independientes, y solo se preocupan por su consumo privado y la oferta total de bien público. Los resultados más importantes de su investigación muestran que solo un pequeño grupo de la sociedad contribuye al bien público y que redistribuciones del ingreso no necesariamente aumentan el número de contribuyentes al bien público. También muestran que los contribuyentes serán aquellos con mayor riqueza, que los contribuyentes van a consumir la 
misma cantidad del bien público como del bien privado y que las contribuciones del Gobierno desplazan las contribuciones privadas.

El modelo propuesto por Bergstrom et al. (1986) es un modelo clásico de altruismo puro. El modelo de Becker (1974) de interacciones sociales, mencionado en la sección anterior, también es un modelo de este tipo. El modelo planteado por Becker analiza dos casos especiales, uno es el de las donaciones y otro el de la redistribución del ingreso entre los miembros de una familia. En el caso de las donaciones, Becker propone que pueden estar motivadas por el deseo de mejorar el bienestar de los destinatarios, por el deseo de evitar el desprecio de los demás o por el deseo de recibir aclamaciones sociales. Este autor encuentra, que la utilidad de los donantes se relaciona de manera positiva con la utilidad de los destinatarios, y que aumentos en los ingresos de los donantes aumentarán sus donaciones.

Explicar las contribuciones voluntarias al bien público desde la teoría económica convencional plantea la necesidad de recurrir a nuevos supuestos. Sugden (1984) afirma que es necesario asumir algún tipo de comportamiento moral que explique por qué las personas se interesan en otras personas. Este autor encuentra que dicho comportamiento es la reciprocidad, en su trabajo propone que las motivaciones recíprocas en un contexto de provisión voluntaria de bienes públicos, puesto que si todos los individuos contribuyen, cada individuo en particular estará en obligación de contribuir al menos lo mismo. En resumen, los modelos mencionados sobre provisión privada de bienes públicos proponen que las donaciones pueden verse como contribuciones privadas al bien público o como provisión de un bien público.

Estas teorías hacen dos aportes importantes al trabajo, en primer lugar, proponen que las donaciones pueden verse como provisión de un bien público y en segundo lugar, que este fenómeno necesita ser estudiado. Entonces: ¿Se puede considerar la elección de conservar la naturaleza en territorios privados como una contribución voluntaria al bien público medio ambiente? ¿Responde la elección de conservar en territorios privados a un deseo por mejorar el bienestar de los demás?

\section{Valores de orientación cultural}

La elección de conservar la naturaleza puede estar motivada por factores diferentes a los propuestos por el análisis económico. Desde la psicología social, Schwartz (2006) formula una teoría que explica los comportamientos y elecciones individuales o grupales. La teoría de Schwartz intenta explicar y caracterizar las culturas o grupo étnicos, de acuerdo con los valores en que enfaticen sus modos de vida, esta se denomina teoría sobre los valores de orientación cultural. Este 
autor plantea que los individuos pertenecientes a una cultura tienden a tomar elecciones y modos de vida de acuerdo con los valores que prevalecen en dicha cultura. El análisis propuesto por este autor se concentra en las culturas, sociedades o grupos étnicos, pero, dicho análisis parte del individuo, por ello su teoría puede servir para entender las motivaciones o valores que influyen en sus decisiones.

Los valores, según Schwartz (2007), son objetos deseables, que transcienden situaciones específicas, que difieren en importancia y que sirven como principios guía en la vida de las personas. Los valores se diferencian unos de otros, por el tipo de meta motivacional que revelan. La teoría sobre los valores de orientación cultural propuesta por este autor, propone que existen diez valores de orientación cultural, cuya relevancia ha sido probada empíricamente en diversos países. Estos diez valores son: el poder, el logro, el hedonismo, la estimulación, la autodirección, el universalismo, la benevolencia, la tradición, la conformidad y la seguridad (en la Tabla 1 se encuentran tales definiciones).

Tabla 1. Definición de los valores de orientación cultural de acuerdo con la meta motivacional que expresan

\begin{tabular}{|l|l|}
\hline \multicolumn{1}{|c|}{ Valor } & \multicolumn{1}{c|}{ Definición } \\
\hline Poder & Estatus social y prestigio, control o dominio sobre las personas y los recursos. \\
\hline Logro & $\begin{array}{l}\text { El éxito personal a través de la demostración de la competencia de acuerdo con } \\
\text { las normas sociales. }\end{array}$ \\
\hline Hedonismo & El placer y la gratificación sensorial para uno mismo. \\
\hline Estimulación & La emoción, la novedad, y el desafío en la vida. \\
\hline Autodirección & El pensamiento independiente y la acción de elegir, crear y explorar. \\
\hline Universalismo & $\begin{array}{l}\text { La comprensión, el aprecio, la tolerancia y la protección del bienestar de todas las } \\
\text { personas y de la naturaleza. }\end{array}$ \\
\hline Benevolencia & $\begin{array}{l}\text { La preservación y el mejoramiento del bienestar de las personas con las que se } \\
\text { está en contacto personal frecuente. }\end{array}$ \\
\hline Tradición & $\begin{array}{l}\text { El respeto por el compromiso y la aceptación de las costumbres e ideas que la } \\
\text { cultura o la religión tradicional prevén para el yo. }\end{array}$ \\
\hline Seguridad & Seguridad, armonía y estabilidad de la sociedad, de las relaciones, y de uno mismo. \\
\hline
\end{tabular}

Fuente: Schwartz (2007, p. 174) traducción libre

Los diez valores de orientación cultural se agrupan en cuatro dimensiones de acuerdo con su dinámica: la automejora, la autotrascendencia, la apertura al cambio y la conservación (Figura 3). La automejora es opuesta a 
la autotrascendencia, la primera enfatiza en el interés propio, mientras que la segunda enfatiza en la preocupación por los demás. En la automejora se agrupan los valores de poder, logro y hedonismo, y en la autotrascendencia se agrupan los valores de universalismo y benevolencia. La búsqueda de un valor puede ir en contra de otros valores, como también puede promover o ser complementada por la búsqueda de valores similares.

Las otras dos dimensiones también esbozan orientaciones opuestas. La apertura al cambio promueve la acción y pensamiento independiente, así como la preparación para las nuevas experiencias, mientras que la conservación promueve la autorrestricción, el orden y la resistencia al cambio. En la apertura al cambio se agrupan los valores de autodirección, estimulación y hedonismo, y en la conservación los valores de seguridad, conformidad y tradición. Schwartz (2007) representa las dimensiones de valores y los valores que agrupan en un esquema circular, en este, mientras más cercanos dos valores en cualquier dirección, más similares sus motivaciones subyacentes, y mientras más lejanos, más opuestas sus motivaciones subyacentes.

Esta teoría propone nuevas alternativas de análisis para la elección de conservar la naturaleza. Cada dimensión puede plantear una alternativa de análisis. La dimensión conservación, en principio podría parecer la más adecuada para analizar dicha elección, puesto que enfatiza en la autorrestricción y la resistencia al cambio. En este sentido, quienes eligen conservar la naturaleza pueden hacerlo motivados bajo la perspectiva de que es un mundo antiguo que se debe conservar. Sin embargo, ¿por qué no considerar otro tipo de motivaciones que pueden plantear las otras tres dimensiones? La dimensión opuesta a la conservación, la dimensión apertura al cambio, enfatiza en la preparación para nuevas experiencias, bajo esta dimensión, la motivación para conservar estaría asociada a la creación de un mundo nuevo por mejorar.

La dimensión autotrascendencia enfatiza en el interés por el bienestar e intereses de los demás. Bajo esta dimensión, la elección de conservar puede ser vista como una acción para mejorar el bienestar de todos. En el análisis opuesto, la dimensión automejora, la elección de conservar puede ser vista como una acción para el bienestar propio, las personas quieren conservar un pedazo del mundo para sí mismos. Estas dos últimas alternativas de análisis son similares a las propuestas por la teoría económica, la automejora puede decirse que se asemeja a la búsqueda de utilidad individual y la autotrascendencia a las motivaciones altruistas y reciprocas. La teoría de orientaciones de valor cultural plantea, entonces, una alternativa de análisis que no contradice el análisis propuesto por la teoría económica, es complementario. 


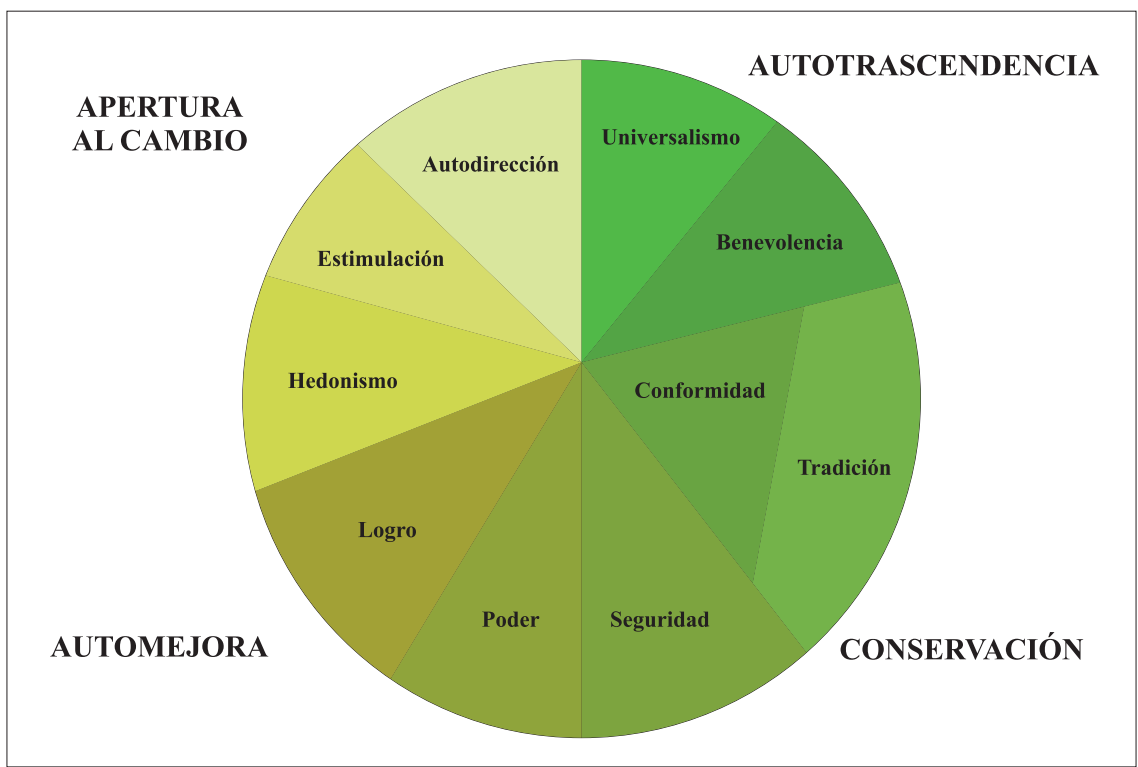

Figura 3. Modelo teórico de las relaciones entre los diez tipos de valores de orientación cultural

Fuente: Schwartz (2007, p. 175), traducción libre.

Ninguna de las teorías o líneas teóricas que recoge el marco teórico plantea una única manera de analizar o entender la decisión de conservar la naturaleza en propiedades privadas. Cada una hace un aporte o brinda luces para el análisis de dicho fenómeno. A raíz de ello, de cada teoría se puede extraer una hipótesis sobre por qué los individuos deciden conservar en sus territorios o cuáles son las repercusiones de esa decisión. Las preguntas formuladas a lo largo de la exposición de las diferentes propuestas teóricas, sirven para formular las hipótesis.

Una primera hipótesis se formula a partir del análisis de la teoría económica de la elección racional: los individuos eligen conservar en sus territorios porque el disfrute de la naturaleza les genera utilidad. Una segunda opción es que la elección de conservar la naturaleza puede verse como una prestación privada del bien público que nace de la preocupación por la naturaleza y el bienestar de la sociedad, y está motivada por el altruismo o la reciprocidad. Una tercera hipótesis, relacionada con la anterior, sería que las personas que eligen conservar en sus propiedades toman esa decisión de acuerdo con unos valores de orientación cultural.

Finalmente hay una cuarta opción que no se puede descartar; los 
incentivos que existen para conservar la naturaleza. En la sección anterior se mencionaron los derechos e incentivos de las RNSC, se nombró que el Estado y algunas ONG ofrecen incentivos para que los individuos conserven la naturaleza en sus propiedades privadas. Entre los incentivos citados se encuentra la exoneración del impuesto predial como el más común. A raíz de ello, una cuarta hipótesis se formula: las personas deciden conservar porque existen incentivos para ello.

\section{METODOLOGÍA}

La investigación tiene un enfoque explicativo y deductivo. Los estudios explicativos, según Hernández, Fernández y Baptista (2003), se caracterizan por buscar las razones o causas que provocan ciertos fenómenos. Por lo general, estos estudios siguen un método deductivo, plantean hipótesis y las contrastan con información empírica. La investigación propuesta indaga por qué conservar áreas naturales de forma privada, plantea unas hipótesis para dicho interrogante a partir de las teorías revisadas en el marco teórico y contrasta las hipótesis con la información empírica obtenida en el trabajo de campo. Se plantea un ejercicio definido, se estudia la conservación en territorios privados en el departamento del Valle del Cauca, y se seleccionan unos casos para ser estudiados y comparados, es decir, se seleccionan Reservas Naturales de la Sociedad Civil en el departamento para ser estudiadas. A continuación se presenta la muestra y el procedimiento de recolección de información.

Para la investigación se seleccionó una muestra de RNSC ubicadas en el Valle del Cauca. La muestra fue seleccionada de acuerdo con el principio de saturación. En este tipo de muestra, según Vásquez et al. (2006), el punto de saturación es alcanzando cuando la información empieza a repetirse, se vuelve redundante y no aporta nuevos aspectos. Por ello, la muestra en este tipo de investigaciones no se construye de forma probabilística, ni debe ser representativa de forma estadística. Los criterios de saturación elegidos son: localización de la reserva, área de la reserva e importancia del municipio donde se encuentra ubicada en términos del número de reservas registradas en ese municipio y el número de hectáreas conservadas en ese municipio.

De acuerdo con el criterio de localización, fueron seleccionadas reservas ubicadas en municipios en el sur, centro y norte del departamento. Bajo el criterio de área, se buscó que estuvieran representadas, tanto reservas de gran tamaño como de tamaño pequeño. En la muestra se encuentran reservas desde 1,87 ha hasta 225,28 ha. Los municipios con mayor número de reservas registradas, como se puede apreciar en la Figura 1, son Versalles y Dagua, por ello se buscó que estos dos municipios estuvieran representados 
en la muestra. Así mismo se buscó que en la muestra estuvieran representadas reservas en los municipios de Tuluá, Cerrito y Dagua, municipios con el mayor número de hectáreas conservadas (ver Figura 2). De acuerdo con los criterios fueron seleccionadas 13 RNSC en los municipios de Cali, Calima El Darién, Dagua, El Cerrito, Tuluá, Versalles y Yumbo. Se buscó que la muestra seleccionada fuera un buen referente de las Reservas Naturales de la Sociedad Civil en el departamento del Valle del Cauca. Además, se buscó que no solo fuera buen referente de la diversidad biológica, sistemas productivos y demás, sino de la diversidad de propietarios, sus profesiones y ocupaciones.

Se eligió la entrevista como instrumento para la recolección de información. Se realizaron entrevistas semiestructuradas, ya que posibilitaban la introducción de preguntas adicionales para obtener mayor información. Fueron realizadas doce entrevistas a los propietarios de las Reservas Naturales de la Sociedad Civil seleccionadas en la muestra (ver el anexo $1^{1}$ ). Para la realización de la entrevista se solicitó a los propietarios entrevistados firmar una autorización para utilizar la información obtenida en la investigación.

En el anexo 2 se encuentra el cuestionario, en la parte inicial del cuestionario se formularon unas preguntas para la caracterización de la reserva; el ecosistema, flora y fauna presentes, los sistemas productivos, entre otros aspectos. Información que sirvió para complementar las características. En la segunda parte se incluyeron las preguntas que indagaban sobre el problema de investigación. Las preguntas que componen la segunda parte del cuestionario fueron elaboradas a partir de las hipótesis propuestas. La guía básica fue el esquema de valores de orientación cultural, que indaga sobre los diez valores de la Tabla 1, en cuatro grupos que confrontan decisiones con inclinación individual o social. Una entrevista basada en este enfoque permite evaluar a la vez la teoría de elección que asume el interés propio como la elección prosocial, porque están incluidas en el enfoque de valores de orientación cultural.

\section{RESULTADOS: MOTIVACIO- NES PARA CONSERVAR LA NATURALEZA EN PROPIE- DADES PRIVADAS}

A partir de las teorías planteadas en el marco teórico, fueron formuladas cuatro hipótesis. A continuación se presenta un análisis con base en la información recogida en el trabajo de campo, donde cada hipótesis se aborda por separado. Inicialmente se trata la hipótesis según la cual las personas deciden

La muestra se compone de 13 RNSC, pero dos pertenecen al mismo propietario, por ello se hicieron 12 entrevistas. 
conservar porque existen incentivos para ello, enseguida se analiza la hipótesis de que los individuos conservan en sus territorios porque les genera utilidad. Posteriormente se estudia la hipótesis de que los individuos conservan por mejorar el bienestar social, por altruismo y reciprocidad, y la provisión privada del bien público. Finalmente se aborda la hipótesis según la cual eligen conservar de acuerdo con unos valores de orientación cultural.

\section{Incentivos para la conservación}

Como una de las hipótesis alternativas para analizar el objeto de este trabajo se planteó que los propietarios de las RNSC conservan porque existen incentivos para hacerlo. Los incentivos y derechos que son otorgados a las RNSC según las leyes y bibliografía revisadas, consisten en derechos de participación en los programas de desarrollo, en derecho al consentimiento previo para la ejecución de inversiones públicas que afecten la reserva y en derecho a incentivos que deberían ser formulados por los distintos municipios. A partir de la información recopilada en las entrevistas se encontró que los municipios en el departamento del Valle del Cauca no han hecho la tarea de crear incentivos para la conservación privada, sin embargo, la CVC, autoridad ambiental en el departamento, y algunas ONG han creado algunos incentivos para conservar en determinados municipios.
El único incentivo que otorgan algunos municipios en el departamento, porque no todos lo hacen, es la rebaja de más o menos un $80 \%$ del impuesto predial. De las 13 reservas entrevistadas, a cinco les rebajan el impuesto predial. Entre las reservas a las que les rebajan el impuesto solo dos afirman que el monto es significativo, ya que ambas cuentan con más de 100 ha, y por ello los impuestos son elevados, estas reservas son; la RNSC Los Chagualos ubicada en el municipio de Tuluá y la RNSC El Vesubio ubicada en el municipio El Cerrito. Para la RNSC El Tesoro, ubicada en el municipio de Dagua, la rebaja no es significativa porque el área es pequeña y los impuestos son bajos, el propietario afirma que los trámites que debe hacer le salen más costosos que el mismo impuesto. Las otras dos reservas a las que les rebajan el impuesto se encuentran ubicadas en el municipio de Versalles, y son la RNSC Villa Manuela y la RNSC La Huerta-Villa Nueva-El Descanso.

A tres reservas les negaron la rebaja del impuesto predial, dos de estas ubicadas en el municipio de Calima y una en el municipio de Yumbo. Las RNSC ubicadas en el municipio de Calima pertenecen al mismo propietario y son RNSC Moralba y RNSC Rondalla. La reserva ubicada en el municipio de Yumbo es la RNSC Parque Ecológico Samuel Álvarez. Las reservas restantes no han solicitado a su respectivo 
municipio la rebaja del impuesto predial o la solicitud está en trámite.

La CVC, en algunos municipios, ha creado incentivos para la conservación privada (CVC, 2012). En Dagua los propietarios entrevistados manifiestan que esta entidad los apoya constantemente, la CVC les ha brindado apoyo y suministros para implementar cercas de aislamiento, para reforestar, para proteger el agua, les ha brindado diversas capacitaciones para la implementación de técnicas sostenibles en sus reservas. En Tuluá, la RNSC Los Chagualos se ha beneficiado de varios proyectos que ha implementado la CVC en la zona para el fortalecimiento de las áreas de conservación. En el resto de municipios en los que se encuentran las reservas de los propietarios entrevistados, la CVC no ha implementado incentivos o los propietarios no se han beneficiado de estos.

En el municipio de Versalles, la ONG Corpoversalles se ha esforzado por crear varios beneficios e incentivos para que propietarios de fincas en la zona conserven. Los propietarios entrevistados cuyas reservas se encuentran en el municipio de Versalles, manifiestan que Corpoversalles les ha brindado educación, capacitación, asistencia técnica. Otra ONG que se esfuerza en la creación de incentivos y beneficios para los propietarios que conservan de forma privada es Resnatur. Los propietarios de la RNSC Refugio Natural Corazones Verdes, ubicada en el municipio de Yumbo, manifiestan que por medio de Resnatur han recibido capacitación en diferentes temas como producción sostenible y agricultura orgánica, la ONG les ha brindado la posibilidad de realizar un inventario de flora y fauna en la reserva que pueda subirse a las bases de datos del Instituto Humboldt, entre otros beneficios e incentivos.

Entre los propietarios entrevistados, algunos manifiestan que aunque no reciben incentivos en el momento, esperan recibirlos en un futuro. Dichos propietarios manifiestan que esperan retribuciones por su labor de conservación y entre las razones por las que conservan, se encuentran los posibles incentivos que puedan recibir, como es el caso de la RNSC La Laguna. Otros propietarios, como el propietario de la RNSC El Vesubio, manifiestan que no conservan porque haya incentivos, puesto que los incentivos que ofrece el Estado son mínimos y tienen otras razones para hacerlo. Sin embargo, todos los propietarios entrevistados, independientemente de las razones por las que conservan, plantean que los incentivos que pueda brindarles el Estado, la CVC, o alguna entidad privada, son bienvenidos.

Retomando la hipótesis formulada, a partir de las entrevistas realizadas se puede afirmar que en algunos casos, entre las motivaciones para conservar, están los incentivos, esto es consistente con otros caso en Latinoamérica 
(Langholz et al., 2000). Como en el caso de las RNSC en Dagua, en el caso de las RNSC en Versalles y en el caso de la RNSC La Laguna en Cali, que esperan recibirlos. Los incentivos que puedan recibir y reciben los propietarios, pesan más en la decisión de registrarse que en la decisión de conservar, porque todos los propietarios de las reservas entrevistados realizaban acciones de conservación antes de efectuar el registro de su predio como RNSC. Muchos propietarios se registraron con la intención de que sus acciones de conservación fueran reconocidas, con la intención de protegerse ante inversiones públicas o proyectos que pudieran afectarlos, con la intención de trabajar en equipo, o con la intención de ser apoyados con incentivos, pero ya efectuaban acciones de conservación.

\section{Conservación por bienestar propio o por la utilidad que confiere la na- turaleza}

Como se planteó en el marco teórico, una de las hipótesis propuestas es que los individuos conservan porque la naturaleza les confiere utilidad. Esta hipótesis, como se argumentó, se complementa o se asemeja a la dimensión automejora de la teoría de valores de orientación cultural. Por ello, en esta sección se aborda la hipótesis conjunta: los individuos conservan por bienestar propio, porque la naturaleza les confiere utilidad y conservan para sí mismos. A partir de las información obtenida en las entrevistas realizadas, se encontró que esta hipótesis es quizás la motivación más fuerte que tienen para conservar los propietarios de las RNSC entrevistados.

Los propietarios de las RNSC El Cedral, San Antonio, El Vesubio, Ronda1la, Moralba, La Laguna, entre otras, manifestaron que conservan porque les apasiona la naturaleza. Disfrutan del espacio que les brinda su reserva, disfrutan de la vegetación presente, de la fauna, del agua, del aire, de los paisajes, entre otros. Para los que viven como los que no viven en la reserva, constituye un espacio de descanso, para algunos constituye un espacio de trabajo, para otros constituye un espacio de recreación. Para el propietario de la RNSC Los Chagualos, como para muchos, la reserva es su proyecto de vida, un lugar donde ve realizadas sus metas y sueños personales, un lugar del que puede disfrutar en el presente y en el futuro, es un espacio de vida. El propietario de la RNSC Tesorito, ubicada en Dagua, manifiesta que su familia siempre ha conservado y que lo hacen por necesidad, porque prevén que si utilizan todo su predio no podrán producir en un futuro, porque si no cuidan el agua no tendrán en épocas de verano, en especial porque viven en una región muy seca.

Quienes tienen producción en sus fincas, han implementado sistemas y técnicas sostenibles, para que en un futuro ellos y sus familias puedan seguir produciendo. Algunos de los que 
producen en sus reservas viven de los recursos generados por esta producción, y por ello son muy conscientes de que deben darle un manejo sostenible, como es el caso de las reservas ubicadas en el municipio de Dagua y en el municipio de Versalles. Para otros propietarios, los recursos generados por la producción hacen parte de los ingresos, como es el caso de la RNSC Los Chagualos, y para otros propietarios, las labores de producción no les generan ingresos para su mantenimiento, pero sí para el sostenimiento de la reserva, como es el caso de la RNSC El Vesubio y la RNSC San Antonio, por lo que también son muy conscientes de que deben producir de forma sostenible.

Los propietarios que no tienen producción sostenible igual son conscientes de que si quieren gozar de ese espacio en un futuro, deben conservarlo. La mayoría de los propietarios manifiestan que conservan porque desean disfrutar de la naturaleza en un futuro, están guardando un pedazo de naturaleza para sí mismos, como también para la comunidad, como es el caso de los propietarios de las RNSC Villa Manuela, San Antonio, Refugio Natural Corazones Verdes, entre otras. Para la mayoría de propietarios poder conservar la naturaleza en sus propiedades es un logro personal, hace parte de sus metas propuestas, de sus proyectos de vida, muchos de estos cuentan con carreras profesionales y técnicas orientadas al medio ambiente.
Las reservas se convierten, entonces, para muchos, en espacios donde aplican sus conocimientos, ya sea para actividades productivas, de ecoturismo, de investigación, de educación, entre otras actividades. En espacios donde disfrutan haciendo lo que les gusta y lo que les apasiona. Por ello, se puede afirmar que quizás la mayor motivación por la que los propietarios conservan es porque les genera utilidad, les genera bienestar propio y porque conservan para sí mismos. El disfrute de la naturaleza y el poder realizar las actividades que los apasiona en sus propiedades, les confiere utilidad a los propietarios de las RNSC. Los distintos sistemas productivos que manejan en sus reservas, las actividades de ecoturismo y educación ambiental, benefician a los propietarios, les brindan recursos para su mantenimiento o disfrute personal. Esta hipótesis propuesta explica en gran medida el por qué los propietarios conservan, sin embargo, existen otras motivaciones relacionadas con las demás hipótesis propuestas.

\section{Conservación por altruismo o reci- procidad}

A partir de las teorías económicas sobre los comportamientos no autointeresados se planteó la hipótesis: los individuos conservan porque les preocupa el bienestar de los demás y de la naturaleza, por motivos altruistas y recíprocos. Posteriormente se planteó que la dimensión autotrascendencia de la teoría de valores de orientación 
cultural plantea un análisis similar que refuerza esta hipótesis, y que por ello se consideraría esta teoría como complementaria a la económica, al analizar la hipótesis expuesta. La teoría sobre los comportamientos no autointeresados plantea que las motivaciones detrás de estos comportamientos son el altruismo y la reciprocidad; por ello, en primer lugar se analizará si la conservación que realizan los propietarios entrevistados es motivada por el altruismo o la reciprocidad.

Ninguno de los propietarios entrevistados conserva por reciprocidad, puesto que ninguno lo hace porque otro propietario esté conservando o porque lo haga el Estado. Solo uno de los propietarios manifiesta explícitamente que conserva por altruismo, que su familia compró los predios donde se encuentran ubicadas la RNSC Rondalla y la RNSC Moralba, exclusivamente para conservar. El propietario de estas reservas argumenta que no tiene intereses lucrativos y que no realiza ninguna actividad en la reserva que le genere ingresos, que conserva porque fue educado en esos valores y porque su familia consideró importante recuperar la cobertura boscosa para proteger los caudales presentes en los predios. Este propietario manifiesta que dado el caso, donaría la propiedad para que el Estado o alguna ONG vele por la conservación en la zona.

El propietario de las RNSC Rondalla y Moralba, y su familia, tienen una fundación que maneja los residuos sólidos, que brinda educación ambiental, que trabaja en proyectos de reforestación y que brinda asesoría en derecho ambiental. De acuerdo con la entrevista realizada a este propietario, se puede vislumbrar que realmente sus motivos son altruistas, así como también, que su nivel económico es superior al de la mayoría de propietarios entrevistados. Ninguno de los demás propietarios entrevistados manifiesta que conserva por motivos altruistas, o por medio de la entrevista se puede intuir que lo haga por ese motivo, sin embargo, todos los entrevistados manifiestan que conservan porque les preocupa el bienestar de la naturaleza y que sus acciones benefician a la comunidad local donde se encuentran ubicadas sus reservas, y en general, a todos.

Muchos de los propietarios proponen que conservan porque les preocupa la fuerte degradación que tienen los ecosistemas y el abastecimiento de agua en el futuro. El propietario de la RNSC El Cedral manifiesta que conserva porque tuvo la oportunidad de ver cómo han cambiado los ecosistemas y cómo se han degradado. Este propietario está convencido de que sus acciones logran cambios positivos en el medio ambiente. Las acciones de conservación, además, tienen impactos positivos en la comunidad y cumplen una función social importante, argumentan varios propietarios. El propietario de la RNSC Los Chagualos, por ejemplo, expone que realiza un fuerte trabajo 
con la comunidad local, que en su reserva brinda capacitaciones para la implementación de técnicas sostenibles en la localidad, y que desde la reserva se formulan proyectos para el desarrollo de la localidad.

Aunque las acciones de conservación de los propietarios favorecen a la comunidad, no se puede afirmar que estos conserven por altruismo o por el deseo de mejorar el bienestar de todos estrictamente. Ninguno de los entrevistados, a excepción del propietario de las RNSC Rondalla y Moralba, estaría dispuesto a donar su predio para que alguna entidad estatal o privada siga conservando y todos los propietarios manifiestan que compartirían su propiedad para que se realicen investigaciones, pero no para ninguna otra actividad. Si la razón por la que conservaran fuera el bienestar social, los propietarios estarían propensos a manifestar que donarían su propiedad, o que la compartirían para muchos usos que no afectaran la conservación

\section{Conservación por valores de orien- tación cultural}

La teoría sobre los valores de orientación cultural propone cuatro enfoques para analizar la elección de conservación de la naturaleza. Dos de los enfoques que propone ya fueron abordados en las hipótesis pasadas como complementarios de las teorías económicas; los individuos conservan por bienestar propio y los individuos conservan para mejorar el bienestar de todos. Respecto al primero de estos enfoques se encontró que la mayoría de los propietarios conservan por bienestar propio, porque buscan su automejora y por utilidad. Se encontró que este enfoque es quizás el que brinda una mejor explicación del por qué los individuos conservan.

En relación con el enfoque: los individuos conservan para mejorar el bienestar de todos, se encontró que la mayoría de propietarios no lo hace por esta razón, solo el propietario de las RNSC Moralba y Rondalla, argumenta tener esta motivación. Los dos enfoques restantes plantean que la elección de conservación puede ser motivada por la visión de que es un mundo antiguo que se debe conservar, o con la visión que es un mundo nuevo por mejorar. La primera perspectiva corresponde a la dimensión de valores denominada conservación y la segunda a la dimensión de valores denominada apertura al cambio. De acuerdo con la información recogida en las entrevistas, la mayoría de los propietarios de las RNSC se inclina por la apertura al cambio, por trabajar por mejorar el mundo actual.

Solo dos de los propietarios entrevistados, el propietario de la RNSC EL Vesubio y el propietario de la RNSC La Laguna, manifestaron que se inclinan por el conservadurismo. Para ambos propietarios el mundo anterior era mejor porque los ecosistemas contaban 
con una menor degradación y la población era menor. Los demás propietarios entrevistados se inclinan por la apertura al cambio, argumentan que en la actualidad se dispone del conocimiento sobre la biodiversidad, sobre la degradación ambiental, y de las tecnologías para prevenir, para prepararnos para el cambio climático, y para implementar técnicas sostenibles. El propietario de la RNSC El Cedral, por ejemplo, propone que todo tiene que evolucionar, que hay que ir con la modernidad, que hay que capacitarse y educarse.

La hipótesis que afirma que los individuos deciden conservar de acuerdo con unos valores de orientación cultural, recoge las múltiples motivaciones y razones detrás de la elección de conservar. Esta hipótesis abarca las dos teorías económicas formuladas y adicionalmente plantea dos enfoques, ofreciendo la visualización de un panorama completo de las razones o motivaciones por las cuales los individuos conservan la naturaleza. No obstante, no debemos dejar de mencionar que los incentivos han sido determinantes para algunos propietarios.

\section{CONCLUSIONES}

Los derechos e incentivos otorgados son un factor determinante en la decisión de registrar el predio y en algunos casos en la decisión de conservar. La mayoría de propietarios manifestó que se registraron como RNSC con la intención de proteger su predio ante inversiones y proyectos públicos o privados que puedan afectarlos, derecho que adquieren al registrarse como RNSC. Estos propietarios también manifestaron que se registraron como RNSC porque buscan apoyo del Estado en sus labores de conservación. Para ellos, los derechos e incentivos fueron determinantes en la decisión de registrarse, pero no en la decisión de conservar, en primer lugar, porque tienen otras motivaciones, y en segundo lugar, porque no hay fuertes incentivos para conservar en el departamento.

Para las RNSC ubicadas en Dagua, Versalles, y Cali, los derechos e incentivos sí son un factor determinante en la decisión de conservar. Aunque los propietarios de estas reservas argumentan que también tienen otras razones para hacerlo, los incentivos que han recibido por parte de la CVC en el caso de las reservas de Dagua, como por parte de Corpoversalles en el caso de las reservas de Versalles, han sido elementos importantes que han influenciado su decisión de conservar y posteriormente de registrarse como RNSC. Dichos incentivos han consistido, en el caso de las reservas en Dagua, en apoyo técnico, capacitación y diversos suministros, y en el caso de las reservas en Versalles, en educación ambiental, capacitación, asistencia técnica, suministros y materiales. En el caso de la RNSC La Laguna ubicada en Cali, el propietario argumenta que entre sus razones para conservar están los posibles incentivos que pueda recibir por 
parte del Estado (conserva porque espera recibir incentivos), pero hasta el momento no ha recibido ninguno.

Conservar la naturaleza por utilidad, por bienestar propio y para sí mismo, es la motivación más fuerte que tienen los propietarios. La mayoría de ellos manifiesta que conserva porque le apasiona la naturaleza y su reserva constituye un espacio donde pueden disfrutar de esta. Las reservas son para algunos sus lugares de trabajo y vivienda, de donde obtienen los recursos para su mantenimiento y el de su familia, por ello, son conscientes que deben proteger el agua, si quieren seguir disfrutando de ella, que deben producir de forma sostenible, si quieren seguir produciendo en un futuro, que deben proteger los bosques, si quieren disfrutar de un aire limpio, y diversidad de especies de flora y fauna. Para quienes no viven, ni trabajan en sus reservas, igual constituye un espacio donde disfrutar, descansar, y aplicar sus conocimientos. La reserva es un logro personal para muchos del que quieren disfrutar por mucho tiempo, es una forma de conservar un pedazo de naturaleza para sí mismos hacia el futuro.

Ninguno de los propietarios entrevistados conserva por motivaciones recíprocas y solo uno conserva por altruismo. El propietario que conserva por altruismo no realiza ninguna actividad en la reserva diferente a conservar, y dado el caso estaría dispuesto a donar la propiedad para que se siga conservando. Cabe resaltar que este propietario tiene un nivel económico superior al de la mayoría de entrevistados. Todos los propietarios argumentan que conservan porque les preocupa el bienestar de los ecosistemas actuales, y que sus acciones benefician a la comunidad, sin embargo, su motivación decisiva es su bienestar propio, en primer lugar. Motivadas por las razones que sean, las acciones de conservación que realizan todos los propietarios benefician a la comunidad local, regional y global, por ello, se puede afirmar que están contribuyendo al bien público medio ambiente.

La dimensión de conservación de la teoría de valores de orientación cultural, que aparentemente parecía ser una fuerte motivación para preservar la naturaleza, no lo es. Solo dos de los propietarios entrevistados manifiestan tener una orientación hacia el conservadurismo. El resto de entrevistados se orientan por la dimensión apertura al cambio, por la evolución y la modernidad, por la preparación ante nuevas experiencias a partir de los conocimientos y tecnologías disponibles. Las otras dos dimensiones, automejora y autotrascendencia, se analizaron en conjunto con las teorías económicas sobre utilidad y sobre reciprocidad y altruismo, respectivamente. La teoría de valores de orientación cultural ofrece un análisis que recoge las diferentes motivaciones y razones que tienen los propietarios para conservar. 
Como se ha argumentado, la elección de conservación de cada propietario es motivada por factores distintos. De las hipótesis propuestas se puede afirmar que aquella que formula que los individuos eligen conservar de acuerdo con unos valores de orientación cultural, es la que mejor explica estas razones, porque abarca varias motivaciones. Entre las motivaciones que abarca se puede afirmar que conservar por bienestar propio es la motivación más fuerte que tienen los propietarios. Los derechos e incentivos influencian más la decisión de registrarse que la decisión de conservar en la mayoría de los casos.

\section{AGRADECIMIENTOS}

A la Corporación Autónoma del Valle del Cauca (CVC), por la información y facilitación de documentos importantes para llevar a buen fin este trabajo. $\mathrm{Y}$ a todos y cada uno de los entrevistados de las Reservas Naturales de la Sociedad Civil, pues sin su valiosa colaboración no hubiese sido posible desarrollar este trabajo.

\section{REFERENCIAS}

Becker, G. (1974). A theory of social interactions. Journal of political economy, 82(6), 1063-1093.

Bergstrom, T., Blume, L. \& Varian, H. (1986). On the private provision of public goods. Journal of Public Economics, 29, 25-49.

Cabrales, A. \& Ponti, G. (2011). Preferencias sociales. En P. Brañas Garza (Ed.), Economía experimental y del comportamiento (pp. 109-124). Barcelona: Antoni Bosh.

Chacón, C.M. \& Solano, P. (2008). La conservación voluntaria por la sociedad civil en América Latina. En C.M. Chacón (Ed.), Voluntad de conservar: experiencias seleccionadas de conservación por la sociedad civil en Iberoamérica (pp. 5-11). San José: The Nature Conservancy y Fundación Biodiversidad.

Presidente de la República (1999, 21 oct.). Decreto 1996. Por el cual se reglamentan los artículos 109 y 110 de la Ley 99 de 1993 sobre reservas naturales de la sociedad civil. Diario Oficial, (43.751).

Congreso de la República. (1999, 22 dic.) Ley 99. Por la cual se crea el Ministerio del Medio Ambiente, se reordena el sector público encargado de la gestión y conservación del medio ambiente y los recursos naturales renovables, se organiza el Sistema Nacional Ambiental, SINA y se dictan otras disposiciones. Diario Oficial, (41.146). 
CVC. (2014a). Listado RNSC. [Archivo de datos]. Cali: CVC.

CVC. (2014b). Folleto informativo reservas naturales de la sociedad civil. Cali: CVC.

CVC. (2012). Reservas naturales de la sociedad civil. Cali: CVC.

Escobar, P. \& Solano, C. (2009). Colombia. En B. Monteferri \& D. Coll (Eds.), Conservación privada y comunitaria en los países amazónicos (pp.109-136). Lima: Sociedad Peruana de Derecho Ambiental.

Fehr, E. \& Fischbacher, U. (2002). Why social preferences matter. The impact of non-selfish motives on competition, cooperation and incentives. Institute for Empirical Research in Economics University of Zurich, Work Paper, (84).

Fehr, E. \& Schmidt, K. (1999). A theory of fairness, competition, and cooperation. The quarterly journal of economics, 114, 817-868.

Field, B. \& Azqueta, D. (1996). Economía y medio ambiente. Bogotá: McGraw-Hill.

Hardin, G. (1968). The tragedy of the commons. Science, 162, 1243-1248.

Hanley, N. \& Barbier, E. (2009). Pricing Nature, Cost-Benefit Analysis and Environmental Policy. Northampton: Edward Elgar.

Hernández, R., Fernández, C. \& Baptista, P. (2003). Metodología de la investigación. México, D.F: McGraw-Hill.

Kolstad, C. (2000). Economía ambiental. México D.F.: Oxford University Press.

Langholz, J., Lassoie, J. \& Schelhas, J. Incentives for Biological Conservation: Costa Rica' Private Wildlife Refuge Program. Conservation Biology, 6, 1735-1743.

Monteferri, B. \& Coll, D. (Eds). (2009). Conservación privada y comunitaria en los países amazónicos. Lima: Sociedad Peruana de Derecho Ambiental.

Ocampo, N. (Ed.). (2010). Mecanismos de conservación privada: una opción viable en Colombia. Bogotá: Grupo Colombiano Interinstitucional de Herramientas de Conservación Privada G5.

Olson, M. (1999). A lógica da ação coletiva: os beneficios públicos e uma teoria dos grupos sociais. São Paulo: Editora da Universidade de São Paulo.

Ostrom, E. et al. (2002). The Drama of the Commons. Washington, D.C.: National Academic Press.

Ostrom, E. (2000). El gobierno de los bienes comunes: la evolución de las instituciones de acción colectiva. México: Fondo de Cultura Económica. 
Parques Nacionales Naturales de Colombia-PNNC- (2014a). Reporte RNSC registradas en el Valle del Cauca. [Archivo de datos]. Colombia: Subdirección de Gestión y Manejo de Áreas Protegidas PNNC.

Parques Nacionales Naturales de Colombia. (2014b). Base de datos RNSC 2001-2011. [Archivo de datos] Recuperado de http:/www.parquesnacionales.gov.co/PNN/ portel/libreria/xls/BasededatosRNSC2001_2011.xls

Parques Nacionales Naturales de Colombia (2014c). Reservas naturales de la sociedad civil. Recuperado de http://www.parquesnacionales.gov.co/PNN/portel/libreria/ php/decide.php?patron $=01.061107$

Pena, J.A. \& Sánchez, J.M. (2006). Altruismo, simpatía y comportamientos prosociales en el análisis económico. Principios, 4, 55-70.

Pindyck, R. \& Rubinfeld, D. (2013). Microeconomía. Madrid: Pearson.

Sugden, R. (1984). Reciprocity: the supply of public goods through voluntary contributions. The Economic Journal, 94, 772-787.

Schwartz, S. (2006). A theory of cultural value orientations: explications and applications. Comparative Sociology, 5, 137-182.

Schwartz, S. (2007). Value orientations: measurement, antecedents, consequences across nations. En R. Jowel, C. Roberts, R. Fitzgerald \& G. Eva (Eds.), Measuring Attitudes Cross-Nationally. Lessons from the European Social Survey (pp. 169-203). London: Sage.

Unidad Administrativa Especial del Sistema de Parques Nacionales Naturales de Colombia, UAESPNN (2001). Política de participación social en la conservación. Bogotá: UAESPNN.

Varian, H. (1993). Análisis microeconómico. Barcelona: Antoni Bosh.

Vásquez, M.L., Ferreira da Silva, M.R., Mogollón A.S., Fernández, M.J., Delgado, E. \& Vargas, I. (2006). Introducción a las técnicas cualitativas en salud. Barcelona: Servei de Publicacions de la Universitat Autònoma de Barcelona.

Zamagni, S. (1995). Economics of altruism. Boloña: Edward Elgar Publishing.

Zamagni, S. (2006). Heterogeneidad motivacional y comportamiento económico. Madrid: Unión Editorial. 
Apuntes CENES Volumen 35, Número 61, ISSN 0120-3053

enero - junio 2016, Págs. 17-48

ANEXOS

Anexo 1: RNSC Seleccionadas para la muestra y propietarios entrevistados

\begin{tabular}{|c|c|c|c|c|}
\hline Nombre RNSC & Municipio & $\begin{array}{c}\text { Corregimiento/ } \\
\text { Vereda }\end{array}$ & Área & $\begin{array}{c}\text { Nombre propietario } \\
\text { entrevistado }\end{array}$ \\
\hline La Laguna & Cali & La Castilla & 1,87 ha & Eusebio Angulo \\
\hline Moralba & Calima El Darín & Berlín & 225,28 ha & Iveth Katheryne Jaramillo \\
\hline Rondalla & Calima El Darín & La Cristalina & 13 ha & Iveth Katheryne Jaramillo \\
\hline El Tesoro & Dagua & Atuncela & 14,96 ha & Oscar Benavidez Saa \\
\hline Tesorito & Dagua & Atuncela & 30,40 ha & Leonel Benítez \\
\hline El Cedral & El Cerrito & El Pomo & 38,4 ha & Alberto Silva Scarpetta \\
\hline El Vesubio & El Cerrito & El Pomo & 138,14 ha & José Genner Gutiérrez \\
\hline San Antonio & El Cerrito & El Pomo & 44,8 ha & Francisco Javier Arango \\
\hline Los Chagualos & Tuluá & El Chuzo y El Brasil & 167,33 ha & María Patricia Mejía \\
\hline Villa Manuela & Versalles & El Balsal & 2,5 ha & Luis Gerardo Monsalve \\
\hline $\begin{array}{c}\text { La Huerta- } \\
\text { Villa Nueva- } \\
\text { El Descanso }\end{array}$ & Versalles & El Balsal & 26,4 ha & Gerardo Antonio Pineda \\
\hline $\begin{array}{c}\text { Parque Ecológico } \\
\text { Samuel Alvarez }\end{array}$ & Yumbo & Dapa & 6,46 ha & Martha Cecilia Cucalón \\
\hline $\begin{array}{c}\text { Refugio Natural } \\
\text { Corazones } \\
\text { Verdes }\end{array}$ & Yumbo & Dapa & 4,74 ha & Perla Barba \\
\hline
\end{tabular}

Anexo 2: Cuestionario de entrevistas. Preguntas sobre la elección de conservación

1. ¿La reserva es propia o familiar?

2. ¿Es su residencia?

3. ¿Considera su reserva un lugar de recreo?

4. ¿A qué se dedica la mayor parte del tiempo?

5. ¿Realiza actividades de investigación en su reserva?

6. ¿Depende de la reserva para su mantenimiento y el de su familia?

7. ¿La reserva le genera ingresos o debe recurrir a otras fuentes para su mantenimiento?

8. ¿Por qué tipo de actividades recibe ingresos (producción sostenible, ecoturismo, educación ambiental)? 
Conservación de la naturaleza en propiedad privada: las Reservas Naturales de la Sociedad Civil en el Valle del Cauca

Melissa Quintero López• Fabio Alberto Arias Arbeláez

9. ¿Recibe apoyo económico de terceros?

10. ¿El Estado lo exonera del impuesto predial?

11. ¿El estado lo exonera del impuesto a las rentas generadas por servicios de ecoturismo?

12. ¿Ha recibido subsidios para el mejoramiento de su vivienda o para la construcción de posadas turísticas en su reserva?

13.¿Ha recibido apoyo económico para la restauración de bosques, ríos, implementación de cercas de aislamiento, entre otras actividades, por parte de la $\mathrm{CVC}$, alguna entidad gubernamental o una ONG?

14. ¿Recibe alguna remuneración económica por servicios ambientales, producción sostenible o alguna otra actividad?

15. ¿Por qué conformo su predio cómo una RNSC?

16. ¿De dónde nace la decisión de conservar?

17.¿Por qué considera importante conservar?

18. ¿Cuáles han sido las dificultades que ha tenido que afrontar y cómo ha logrado superarlas?

19. ¿La reserva es una forma de asegurar una parte de la naturaleza a futuro?

20. ¿Le gustaría que su familia continuara con su labor de conservación?

21.¿Considera que su labor es buena para la sociedad?

22. ¿Es admirado por su labor?

23. ¿Considera un logro personal su labor?

24. ¿Compartiría la propiedad para ejecutar acciones encaminadas a la conservación y manejo sostenible?

25. ¿Donaría su propiedad al estado o a una ONG para que realizará acciones de investigación, conservación y manejo sostenible?

26.¿Cuál es su percepción del estado de los ecosistemas actuales?

27. Desde su experiencia como protector de la naturaleza y de vida ¿A qué le apostaría usted para detener la degradación?

28. ¿Conservar es su forma de contribuir al mundo?

29. ¿Considera que el mundo anterior era mejor y hay que volver a él?

30. ¿Cómo relaciona la conservación con la comunidad?

31.¿Qué tanto le preocupa el bienestar de la sociedad? 